\title{
Erratum: Oxidation and emission of methane in a monomictic lake (Rotsee, Switzerland)
}

\author{
C. J. Schubert • F. S. Lucas • E. Durisch-Kaiser • \\ R. Stierli $\cdot$ T. Diem $\cdot$ O. Scheidegger $\cdot$ F. Vazquez \\ B. Müller
}

Published online: 14 December 2010

(C) Springer Basel AG 2010

Erratum to: Aquat. Sci. (2010) 72:455-466

DOI 10.1007/s00027-010-0148-5

On page 457, Eq. 3: "c" should be an exponent.

On page 464: we stated that $1,700 \mathrm{t}$ methane could be released during turnover. This number should be $10 \mathrm{t}$ of methane.

The online version of the original article can be found under doi:10.1007/s00027-010-0148-5.

Present Address:

F. S. Lucas

Faculté des Sciences et Technologie, Cereve UMR MA 102,

Université Paris 12, 61 ave du General de Gaulle,

94010 Creteil Cedex, France

Present Address:

E. Durisch-Kaiser

Institute of Biogeochemistry and Pollutant Dynamics,

Swiss Federal Institute of Technology (ETH),

Universitätsstrasse 16, 8092 Zurich, Switzerland

C. J. Schubert $(\varangle) \cdot$ F. S. Lucas · E. Durisch-Kaiser •

R. Stierli · T. Diem · O. Scheidegger · F. Vazquez · B. Müller

Department of Surface Waters-Research and Management,

Eawag, Swiss Federal Institute of Aquatic Science and

Technology, 6047 Kastanienbaum, Switzerland

e-mail: carsten.schubert@eawag.ch 